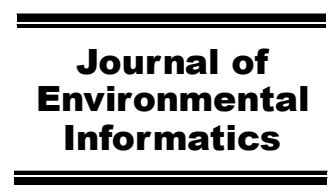

www.iseis.org/jei

\title{
Uncertainty Assessment of Non-normal Emission Estimates Using Non-Parametric Bootstrap Confidence Intervals
}

\author{
L. I. Tong*, R. Saminathan*, and C. W. Chang \\ Department of Industrial Engineering and Management, National Chiao Tung University, Hsinchu 300, Taiwan
}

Received 16 January 2014; revised 27 April 2015; accepted 4 May 2015; published online 9 December 2015

\begin{abstract}
Mitigating global warming problems initially involves reducing greenhouse gas (GHG) emissions, therefore the uncertainty of GHG emission estimates needs to be assessed concisely. Although the uncertainty of GHG emission estimates is generally evaluated using classical confidence interval, quantifying the uncertainty based on non-normal GHG emission estimates or small dataset may lead to a significant bias. Using bootstrap confidence intervals is an effective means of reducing such a bias. This study presents a procedure for constructing four bootstrap confidence intervals to assess the uncertainty of GHG emission estimates for three non-normal distributions (namely, Weibull, Gamma and Beta). These bootstrap confidence intervals are standard bootstrap (SB) confidence interval, percentile bootstrap (PB) confidence interval, Bias-corrected percentiles bootstrap (BCPB) confidence interval and bias-corrected and accelerated (BCa) confidence interval. The sensitivity of bootstrap intervals for emission data is examined under various combinations of sample size and parameters of underlying non-normal distributions using three indices: coverage performance, interval mean, and interval standard deviation. Simulation results indicate that the bootstrap confidence intervals for assessing the uncertainty of emission estimates are highly applicable with small sample size and the data distribution is non-normal. Compared with the classical confidence interval, bootstrap confidence intervals have smaller interval mean and smaller interval standard deviation for small sample size under non-normal distributions. This study recommends BCa confidence interval to assess the uncertainty of the emission estimates as long as sample size is 15 or more and the distribution is non-normal. A case study with emission data of the High-Bleed Pneumatic controllers demonstrates the effectiveness of the proposed procedure
\end{abstract}

Keywords: bootstrap confidence intervals, greenhouse gases, emissions, non-normal distributions, uncertainties

\section{Introduction}

Human activities in recent decades have dramatically influenced the atmospheric changes in terms of the amount of greenhouse gases (GHG). Global warming is caused by the increasing GHG emissions emitted into the atmosphere. Global warming have the heterogeneous effects on the nations in the world; these effects will include changes in rainfall patterns, changes in agricultural yields, the continued melting of glaciers and species extinctions, increase of coastal erosion, seasonal changes in some regions, and emergence of new infectious diseases (IPCC, 2007; Kumar et al., 2012; Pinto et al., 2008; Riebeek, 2010). These impacts have alerted scientists worldwide to act aggressively in controlling GHG emissions. Numerous studies have attempted to reduce GHG emissions and their effects (e.g. Erickson et al., 2012; Millar et al., 2012; Shen et al., 2012;

${ }^{*}$ Corresponding author. Tel.: +886-3-5733873; fax: +886-3-5731896. E-mail address: litong@cc.nctu.edu.tw (L. I. Tong).

* Corresponding author. Tel.: +91-9944382772; fax: +886-3-5731896. E-mail address: sami2k50@yahoo.co.in (R. Saminathan).

ISSN: 1726-2135 print/1684-8799 online

(C) 2016 ISEIS All rights reserved. doi:10.3808/jei.201500322
Mudhoo et al., 2013; Watson et al., 2013). The Kyoto Protocol was developed by United Nations Framework Convention on Climate Change to reduce GHG emissions (UNFCCC, 1997). Notably, implementing the Kyoto protocol requires high quality GHG inventory data. However, inventory uncertainties, even when prepared according to Intergovernmental Panel on Climate Change (IPCC) guidelines and good practice, are high for inventory applications (IPCC, 2006). Random errors and biases in emission inventories may lead to erroneous conclusions on emission trends, source apportionment, and compliance of emission (Frey et al., 1999). Uncertainty information is essential for characterizing the quality of emission inventory. The quality of the GHG inventory can be improved by accurately quantifying the uncertainty of the emission estimates.

Emission estimates are made by multiplying an appropriate emission factor with activity data. The two major types of uncertainty associated with emission estimates are model uncertainty and parameter uncertainty (GHG Protocol, 2003; IPCC, 2006; Rypdal and Winiwarter, 2001). Model uncertainty refers to the uncertainty associated with the models (i.e. mathematical equations) which characterize the relationships between various parameters (or inputs such as e.g. activity data, emission factors or other parameters) and emission processes (GHG Protocol, 2003). Model uncertainty arises when the incorrect ma- 
thematical model or inappropriate parameters (i.e. inputs) in the model were used (GHG Protocol, 2003; IPCC, 2006; Rypdal and Winiwarter, 2001). Quantifying model uncertainty of the emission esti- mates is difficult in practice, owing to the complexity in developing appropriate estimation models (IPCC, 2006). Parameter uncertainty refers to the uncertainty associated with quantifying the parameters used as inputs in the estimation model. Parameter uncertainty arises owing to lack of precise inputs (GHG Protocol, 2003; IPCC, 2006). Parameter uncertainties can be evaluated through statistical analysis and expert judgment (IPCC, 2001). Parameter uncertainty is the primary type and source of uncertainty associated with emission estimates (GHG Protocol, 2003; IPCC, 2006). Therefore, quantifying parameter of emission estimates is often considered when investigating the uncertainty in the emission inventories.

According to IPCC guidelines, the uncertainties are generally expressed in the uncertainty range which is described by $95 \%$ confidence interval within which the underlying value of an uncertainty quantity is thought to lie (IPCC, 2006; Zhao et al., 2011; EMEP/EEA, 2013). Classical methods, expert judgment and utilization recommendations were recommended by IPCC to express the uncertainty of GHG emission estimates (IPCC, 2006). Classical methods (e.g., Maximum Likelihood Estimation, Least Square Estimation, Method of Moments and $95 \%$ confidence interval) require sufficiently large data to estimate the uncertainty and the distribution of data must be normal as well. When data is lacking, the uncertainty is estimated based on expert judgment. Utilization recommendations can be adopted when both classical methods and expert judgment fail. When the sample size is small and data distribution is not normal for emission estimates, constructing a 95\% confidence interval to quantify the uncertainty of emission estimates using classical methods is inappropriate. In this case, bootstrap method can be utilized as an alternative approach to the classical method. The bootstrap method is a computationally intensive statistical approach to make inferences about population based on small dataset without making distributional assumptions.

The bootstrap method has two different types which are referred as the "non-parametric" and "parametric" approaches (Efron and Tibshirani, 1993; Kyselý, 2010). The parametric bootstrap method generates the bootstrap observations by a parametric distribution. In parametric bootstrap method, a specified probability distribution is fitted to the original dataset in advance. The non-parametric bootstrap method resamples the observations from original sample. Studies based on bootstrap method have attempted to quantify the uncertainty in emission factors and inventories (Frey and Bammi, 2002; Frey et al., 1999; Frey and Rhodes, 1996a; Frey and Zhao, 2004; Rhodes and Frey, 1997). In the case of an uncensored dataset, confidence intervals constructed using parametric bootstrap simulation was presented to quantify the uncertainty of emission estimates (Frey and Rhodes, 1996b). Similar studies were attempted on an emission dataset from lean burn engines and censored mercury emission factor dataset to quantify the uncertainty of emission estimates (Frey and Li, 2003; Zhao and Frey, 2003; Zhao and Frey, 2006). Tong et al. (2012) constructed confidence intervals using the non-parametric bootstrap method to quan- tify the uncertainty of GHG emission estimates under normal, log-normal and uniform distributions (Tong et al., 2012). Their results suggested sample size greater than or equal to 9 for estimating the uncertainty of emission estimates using bootstrap confidence intervals. In real situation, most of emission estimates cannot be adequately described by the normal distribution. However, their study considered only two non-normal distributions (log-normal and uniform). The log-normal distribution is a tail-heavy distribution and is used when the uncertainties of emission estimates are expected to be positively skewed. The uniform distribution is used to describe an equal likelihood of obtaining any value within a range. It is often utilized to represent physically-bounded quantities such as fraction that must vary between 0 and 1 (Rhodes and Frey, 1997; EMEP/EEA, 2013). These two non-normal distributions may not cover most of the skewed distributions. The Weibull and Gamma have approximately similar properties to log-normal but are less tail-heavy than log-normal. The Weibull distribution is generally used for describing highly asymmetrical uncertainties of emission estimates (Monni and Syri, 2004). The Gamma distribution is useful in describing large and highly skewed uncertainties of emission estimates. The Beta distribution is defined on the continuum between 0 and 1 and can be either positively or negatively skewed. It is used mostly in cases where the value used in the inventory seems to be too high (Monni and Syri, 2004). Therefore, this study considers these three skewed distributions (i.e., Weibull, Gamma and Beta) to obtain more general and comprehensive conclusions, when using the non-parametric bootstrap method.

This study proposes a procedure to quantify the uncertainty of emission estimates using bootstrap resampling method and four bootstrap confidence intervals. Based on the three abovementioned non-normal distributions with different parameter combinations, non-normal cases are simulated to meet realworld situations. The performance of the proposed bootstrap confidence intervals is evaluated using three indices (i.e. coverage performance, interval mean and interval standard deviation). Furthermore, this study attempts to find an appropriate sample size when constructing bootstrap confidence intervals for quantifying uncertainty of emission estimates.

The rest of this paper is organized as follows. Section 2 introduces the concept of bootstrap method and bootstrap confidence intervals. Section 3 simulation procedure for estimating the uncertainty of non-normal GHG emission estimates using bootstrap confidence intervals. Section 4 summarizes the results of sensitivity analysis of bootstrap confidence intervals to demonstrate the effectiveness of the proposed method. Section 5 describes a case study to demonstrate the effectiveness of the bootstrap construct a confidence interval for the emission estimates. Conclusion is finally drawn in Section 6.

\section{Concept of Bootstrap Method and Bootstrap Con- fidence Intervals}

Efron introduced the bootstrap method which is a databased simulation method for statistical inference (Efron, 1979). In particular, the bootstrap method relies on resampling with 
replacement from the given sample and calculating the required statistic from these repeated samples. The values of the statistic from the repeated sampling can then be used to generate standard errors and confidence intervals for the statistic. Advantageously, bootstrap method does not rely on distributional assumptions about the underlying population.

$\operatorname{Suppose}\left(x_{1}, x_{2}, \ldots, x_{n}\right)$ is an original sample of size $\mathrm{n}$ randomly taken from a process. Let $\left(x_{1}^{*}, x_{2}^{*}, \ldots, x_{n}^{*}\right)$ be a bootstrap sample of size $\mathrm{n}$ drawn (with replacement) from the original sample. Hence, a total of $n^{n}$ resamples are possible. Bootstrap sampling is equivalent to sampling with replacement from the empirical population distribution. The bootstrap estimate is calculated for each bootstrap sample, and the subsequent empirical distribution is referred to as the bootstrap distribution of the statistic. At least 1000 bootstrap resamples are recommended as sufficient to obtain reasonably accurate confidence interval estimates for the parameter (Efron and Tibshirani, 1993).

Assume that the population parameter $\theta$ is estimated using a random sample. The bootstrap estimate of $\theta$ is represented by $\hat{\theta}^{*}$. The resampling procedure is repeated $\mathrm{B}$ times, where $B \geq$ 1000 . The $B$ bootstrap estimates $\hat{\theta}_{1}^{*}, \hat{\theta}_{2}^{*}, \ldots, \hat{\theta}_{B}^{*}$ can be calculated from $B$ resamples. Four bootstrap confidence intervals are the standard bootstrap (SB) confidence interval, percentile bootstrap (PB) confidence interval, bias-corrected percentile bootstrap (BCPB) confidence interval and bias-corrected accelerated percentile bootstrap $(\mathrm{BCa})$ confidence interval, as developed by Efron (Efron, 1981; Efron, 1982), Efron \& Gong (Efron and Gong, 1983), Efron \& Tibshirani (Efron and Tibshirani, 1986), and Efron (Efron, 1987). The formulas used to calculate these intervals are detailed below:

\section{SB confidence interval}

The mean of B bootstrap estimates $\hat{\theta}_{i}^{*}(i=1,2, \ldots, B)$ can be computed as follows:

$$
\hat{\theta}^{*}=\frac{1}{B} \sum_{i=1}^{B} \hat{\theta}_{i}^{*}
$$

The standard deviation of B bootstrap estimates $\hat{\theta}_{i}^{*} \quad(i=1$, $2, \ldots$, B) can be computed as follows:

$\hat{S}_{\hat{\theta}^{*}}=\left[\left(\sum_{i=1}^{B}\left[\hat{\theta}_{i}^{*}-\hat{\theta}^{*}\right]^{2}\right) /(B-1)\right]^{1 / 2}$

when the distribution of $\hat{\theta}^{*}$ is approximately normal, the (1 $\alpha) 100 \%$ SB bootstrap confidence interval for $\theta$ can be computed as $\left(\hat{\theta}^{*}-z_{(1-\alpha / 2)} \hat{S}_{\hat{\theta}^{*}}, \hat{\theta}^{*}+z_{(1-\alpha / 2)} \hat{S}_{\hat{\theta}^{*}}\right)$, where, $z_{(1-\alpha / 2)}$ is the $(1-\alpha / 2)^{\text {th }}$ percentile of the standard normal distribution. Despite the relative ease in calculating the SB confidence interval, normality assumption is required on the bootstrap distribution of $\hat{\theta}_{i}^{*}$ (Efron, 1982).

\section{PB confidence interval}

PB method is also called bootstrap-p method. By ascending the $\hat{\theta}_{i}^{*}(i=1,2, \ldots, B)$, an ordered estimate $\hat{\theta}^{*}(i)(i=1$, $2, \ldots, B)$ can be obtained, of which the $(\alpha / 2)^{\text {th }}$ percentile and the $(1-\alpha / 2)^{\text {th }}$ percentile are the left and right endpoints of the confidence interval for $\theta$, respectively. The $(1-\alpha) 100 \%$ PB confidence interval for $\theta$ can be computed as follows:

$\left(\hat{\theta}_{((\alpha / 2) B)}^{*}, \hat{\theta}_{((1-\alpha / 2) B)}^{*}\right)$

If the distribution of $\hat{\theta}^{*}$ is not normal, the PB confidence interval is preferable (Moore et al., 2008).

\section{BCPB confidence interval}

The bootstrap empirical distribution may occasionally be asymmetric, resulting in a bias in the confidence interval. The PB confidence interval is thus corrected (Efron, 1981).

First, $P_{0}$ is computed using the bootstrap distribution of $\hat{\theta}^{*}(i)(i=1,2, \ldots, B)$ as follows:

$P_{0}=\operatorname{Pr}\left(\hat{\theta}^{*}(i) \leq \hat{\theta}\right),(i=1,2, \ldots, B)$

Next, $z_{0}$ is computed to measure the bias of bootstrap distribution as follows:

$z_{0}=\Phi^{-1}\left(P_{0}\right)$

where $\Phi^{-1}($.$) is the inverse cumulative standard normal distri-$ bution.

The bias-corrected percentiles $P_{L}$ and $P_{U}$ are computed, respectively, as given in the following formulas:

$P_{L}=\Phi\left(2 z_{0}-z_{1-\alpha / 2}\right)$

$P_{U}=\Phi\left(2 z_{0}+z_{1-\alpha / 2}\right)$

where $\Phi($.$) is the cumulative standard normal distribution.$ Thus, the $(1-\alpha) 100 \%$ BCPB confidence interval for $\theta$ can be computed as $\left(\hat{\theta}^{*}\left(P_{L} B\right), \hat{\theta}^{*}\left(P_{U} B\right)\right)$.

\section{4. $\mathrm{BCa}$ confidence interval}

As an improved version of $\mathrm{PB}$ confidence interval, the $\mathrm{BCa}$ confidence interval can accelerate the correction of the estimated error of biased data in the PB confidence interval (Efron, 1982). The $P_{L}$ and $P_{U}$ in $\mathrm{PB}$ confidence intervals are revised as

$$
\begin{aligned}
& P_{L}=\Phi\left(z_{0}+\frac{z_{0}+z_{\alpha}}{1-a\left(z_{0}+z_{\alpha}\right)}\right) \\
& P_{U}=\Phi\left(z_{0}+\frac{z_{0}+z_{1-\alpha}}{1-a\left(z_{0}+z_{1-\alpha}\right)}\right)
\end{aligned}
$$

where $a=\sum_{i=1}^{B}\left(\hat{\theta}^{*}-\hat{\theta}^{*}(i)\right)^{3} / 6\left[\sum_{i=1}^{B}\left(\hat{\theta}^{*}-\hat{\theta}^{*}(i)\right)^{2}\right]^{3 / 2}$. 

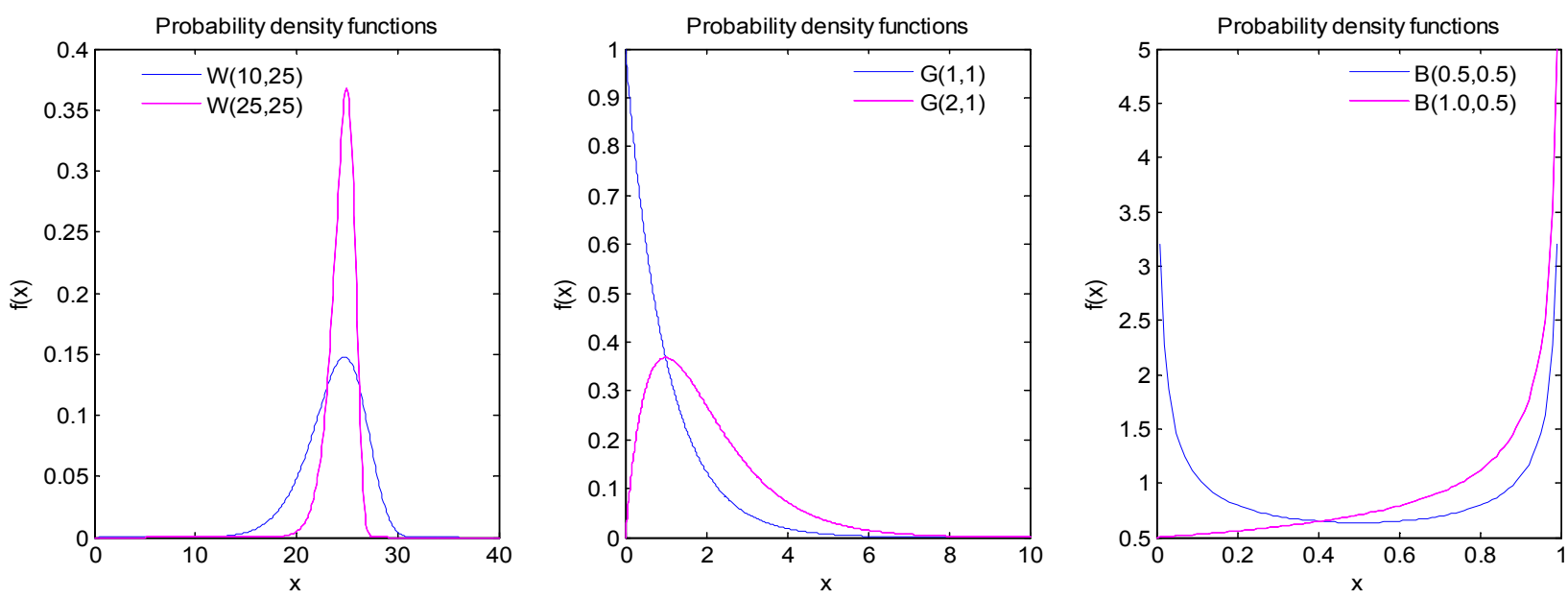

Figure 1. Density Plots for various combinations of parameters $(\alpha, \beta)$ of Weibull, Gamma and Beta distributions.

Table 1. The Parameters of Non-normal Distributions Weibull, Gamma and Lognormal

\begin{tabular}{llll}
\hline Name of Distribution & Parameters & Mean & Standard Deviation \\
\hline Weibull $(\alpha, \beta)$ & $\alpha=10, \beta=25$ & 23.7838 & 8.1878 \\
& $\alpha=25, \beta=25$ & 24.4610 & 1.4900 \\
$\operatorname{Gamma}(\alpha, \beta)$ & $\alpha=2, \beta=1$ & 2 & 1.4142 \\
$\operatorname{Beta}(\alpha, \beta)$ & $\alpha=1, \beta=1$ & 1 & 1 \\
& $\alpha=0.5, \beta=0.5$ & 0.5 & 0.1250 \\
& $\alpha=1.0, \beta=0.5$ & 0.6667 & 0.0889 \\
\hline
\end{tabular}

Here, $z_{0}$ and $a$ are the bias-correction and acceleration constants, respectively. Thus, the $(1-\alpha) 100 \%$ BCa confidence interval for $\theta$ is obtained as $\left(\hat{\theta}^{*}\left(P_{L} B\right), \hat{\theta}^{*}\left(P_{U} B\right)\right)$. Notably, if $z_{0}$ and $a$ are zero, then the $\mathrm{BCa}$ confidence interval is the same as the PB confidence interval. Efron and Tibshirani (Efron and Tibshirani, 1993) recommended the BCa confidence intervals for general use, despite the lack of a standard rule for identifying which method has the best confident interval (Zheng, 2002).

\section{Simulation Procedure for Estimating the Uncer- tainty of Non-normal GHG Emission Estimates Us- ing Bootstrap Confidence Intervals}

The sample size of emission data obtained is generally small. In this case, the classical $95 \%$ confidence interval may lead to a significant bias, if the distribution of emission data is nonnormal. The bootstrap method is an alternative approach to the classical method. The bootstrap method has two different types which are "non-parametric" and "parametric" approaches. In parametric bootstrap method, a probability distribution is fitted to the original dataset in advance. However, fitting a distribution to emission data of small sample size is difficult. Moreover, the estimates of the parameters of the fitted distribution may have large estimation error. Thus, the parametric bootstrap method is sometimes not applicable. Non-parametric bootstrap method can be utilized to quantify the uncertainty of emission estimates. The procedure of estimating the uncertainty of GHG emission estimates using non-parametric bootstrap method is described as follows. Collect a set of emission data $\left(x_{1}, x_{2}, \ldots\right.$, $x_{n}$ ) (Original sample). Generate a total of $\mathrm{B}(\mathrm{B} \geq 1000)$ bootstrap resample $\left(x_{1}^{*}, x_{2}^{*}, \ldots, x_{n_{k}}^{*}\right)$ from the original sample with replacement and compute $\bar{x}_{i}^{*}$ for each bootstrap resample $(i=$ $1,2, \ldots$, B). Construct the $(1-\alpha) 100 \%$ bootstrap confidence intervals for the true mean of emission estimates.

To verify the effectiveness of quantifying uncertainty of GHG emission estimates using non-parametric bootstrap method, three non-normal distributions (namely, Weibull, Gamma and Beta) were considered in this study. These distributions are generally used to represent the variability in probabilistic assessment regarding estimation of the uncertainty of emission estimates (IPCC, 2006; EMEP/EEA, 2013). Therefore, these distributions were used to generate population emission data. The simulation procedure for analyzing the accuracy and sensitivity of the bootstrap confidence interval with various combinations of sample size and parameters under three non-normal distributions is described as follows. The combinations of parameters are presented in Table 1. In Table 1, $\alpha$ represents the shape parameter and $\beta$ represents the scale parameter.

(i) Generate emission data using Monte Carlo method by choosing a parameter set for each non-normal distribution in Table 1;

(ii) Collect a random sample of size $n\left(x_{1}, x_{2}, \ldots, x_{n}\right)$ from the simulated data (original sample);

(iii) Generate bootstrap resample dataset $B=1000$ times (with replacement) from the original sample each with size $n$ 
$\left(x_{1}^{*}, x_{2}^{*}, \ldots, x_{n}^{*}\right)$ B);

(iv) Compute the mean $\bar{x}_{i}^{*}$ from $n$ sample $(i=1,2, \ldots$,

(v) Construct $(1-\alpha) 100 \%$ confidence interval for the true mean of emission estimates by using the four Bootstrap methods; and

(vi) Repeat (ii) - (v) $N=2000$ times to obtain sets of bootstrap confidence intervals. With these $N$ intervals, the coverage performance index, interval mean and standard deviation are calculated for sensitivity analysis of bootstrap intervals.

The above simulation procedure was repeated for each set of parameters with various sample size $n(n=5,6, \ldots, 30)$ for each of the non-normal distributions. These distributions can have various shapes (such as skewed, very flat or steep) depending on their parameters. For having a general conclusion of non-normal distributions, this study considers parameter values for the most extreme cases of non-normal distributions. Figure 1 shows the density plots of the assumed distributions for various values of parameters $(\alpha, \beta)$ in Table 1 .

The performance of four bootstrap confidence intervals for GHG emission estimates was evaluated using the three indices (Chou et al., 2006; Tong et al., 2008; Tong et al., 2012):

1. Coverage performance index: This index represents the percentage of times that the actual emission falls into the bootstrap confidence intervals. A larger performance index value implies more accurate bootstrap confidence interval estimates.

2. Interval mean index: This index represents the average of interval lengths (i.e. difference between the lower and upper limits) of $\mathrm{N}$ bootstrap confidence intervals. Moreover, a smaller value of interval mean index implies a more precise estimation and improved performance of the bootstrap confidence interval estimates. The appearance of smaller interval means is associated with a smaller coverage performance, implying a trade-off between precision and accuracy.

3. Interval standard deviation index: This index represents the standard deviation of interval lengths of $\mathrm{N}$ bootstrap confidence intervals. A small standard deviation implies a smaller estimated variation and improved performance of bootstrap confidence interval estimates.

The coverage probability of the confidence interval for a parameter is close to a nominal value of 0.95 under normal distribution (Kyselý, 2010). For non-normal distribution, the coverage probability of the confidence interval for a parameter can be appreciably below 0.95 (Niwitpong and Kirdwichai, 2008). The coverage performance with short average interval length in all situations is the evidence for good bootstrap confidence intervals (Chu and Ke, 2006; Efron and Tibshirani, 1986). The $95 \%$ confidence interval using classical method of emission estimates were calculated for comparison with bootstrap confidence intervals using the similar procedure. Hereafter, the $95 \%$ confidence interval is referred as the "classical confidence interval. Matlab software was used to generate the dataset of emission estimates and construct confidence intervals along with the sensitivity analysis.

\section{Result of Sensitivity Analysis}

Results of simulation procedure are presented as follows. Three performance indices were plotted against various sample size $\mathrm{n}$ for each non-normal distribution (i.e., Weibull, Gamma and Beta) (Figures $2 \sim 4$ ). In those figures, respective abbreviations of classical confidence interval, standard bootstrap, percentile bootstrap, biased-corrected percentile bootstrap and bias-corrected accelerated were represented as Classical, SB, $\mathrm{PB}, \mathrm{BCPB}$ and $\mathrm{BCa}$. The scale difference in vertical axis of Figures $2 \sim 4$ arises owing to use of different parameter values. An increase in sample size $n(n=5,6, \ldots, 30)$ gives larger coverage performance, while both interval mean and standard deviation decreases. Thus, improvement of estimation precision and accuracy for the four bootstrap confidence intervals and the classical confidence interval depends on the increased sample size.

Figure 2a indicates that when the sample size $n$ exceeds 15 for Weibull $(\alpha=10, \beta=25)$ and 12 for Weibull $(\alpha=25, \beta=25)$, the coverage performance is approximately 0.90 or above. Figure $3 \mathrm{a}$ indicates that when the sample size $n$ exceeds 16 for both $\operatorname{Gamma}(\alpha=1.0, \beta=1.0)$ and $\operatorname{Gamma}(\alpha=2.0, \beta=1.0)$, the coverage performance is approximately 0.90 or above. Figure $4 \mathrm{a}$ indicates that when the sample size $n$ exceeds 8 for $\operatorname{Beta}(\alpha=0.5, \beta=0.5)$ and 16 for $\operatorname{Beta}(\alpha=1.0, \beta=0.5)$, the coverage performance is approximately 0.90 or above. Although all four methods display a similar trend in terms of coverage performance, the BCa method largely outperforms the SB, PB and $\mathrm{BCPB}$ methods for all three non-normal (i.e. Weibull, Gamma and Beta) distributed emission estimates. The small differences in results when comparing these methods are of little practical significance in the cases explored here. For non-normal cases, the coverage performances of classical confidence interval are lower than $95 \%$ when the sample size is small.

The four bootstrap confidence intervals were compared using interval mean and interval standard deviation. Figures $2 \mathrm{~b}$, $3 \mathrm{~b}$ and $4 \mathrm{~b}$ indicate that all interval means for the four bootstrap confidence intervals decrease with sample size $n$. The differences among the average interval lengths of the four bootstrap confidence intervals are negligible when sample size $n$ is greater than 15 . Figures $2 \mathrm{c}, 3 \mathrm{c}$ and $4 \mathrm{c}$ indicate that all interval standard deviations for the four bootstrap confidence intervals decrease with sample size $n$. The differences among the interval standard deviations of the four bootstrap confidence intervals are negligible when the sample size $\mathrm{n}$ is greater than 15 . For non-normal cases, the average interval lengths and interval standard deviations of classical confidence interval are higher than four bootstrap confidence intervals when the sample size is small and are quite similar to bootstrap confidence when sample size is greater than 15 .

The bootstrap confidence intervals for the emission estimates were compared to the results from the classical confidence interval and also among themselves. The comparison results are summarized as follows. Bootstrap confidence intervals perform better than classical confidence interval for small dataset under non-normal situation in terms of interval mean and interval standard deviation. BCa confidence interval performs better 

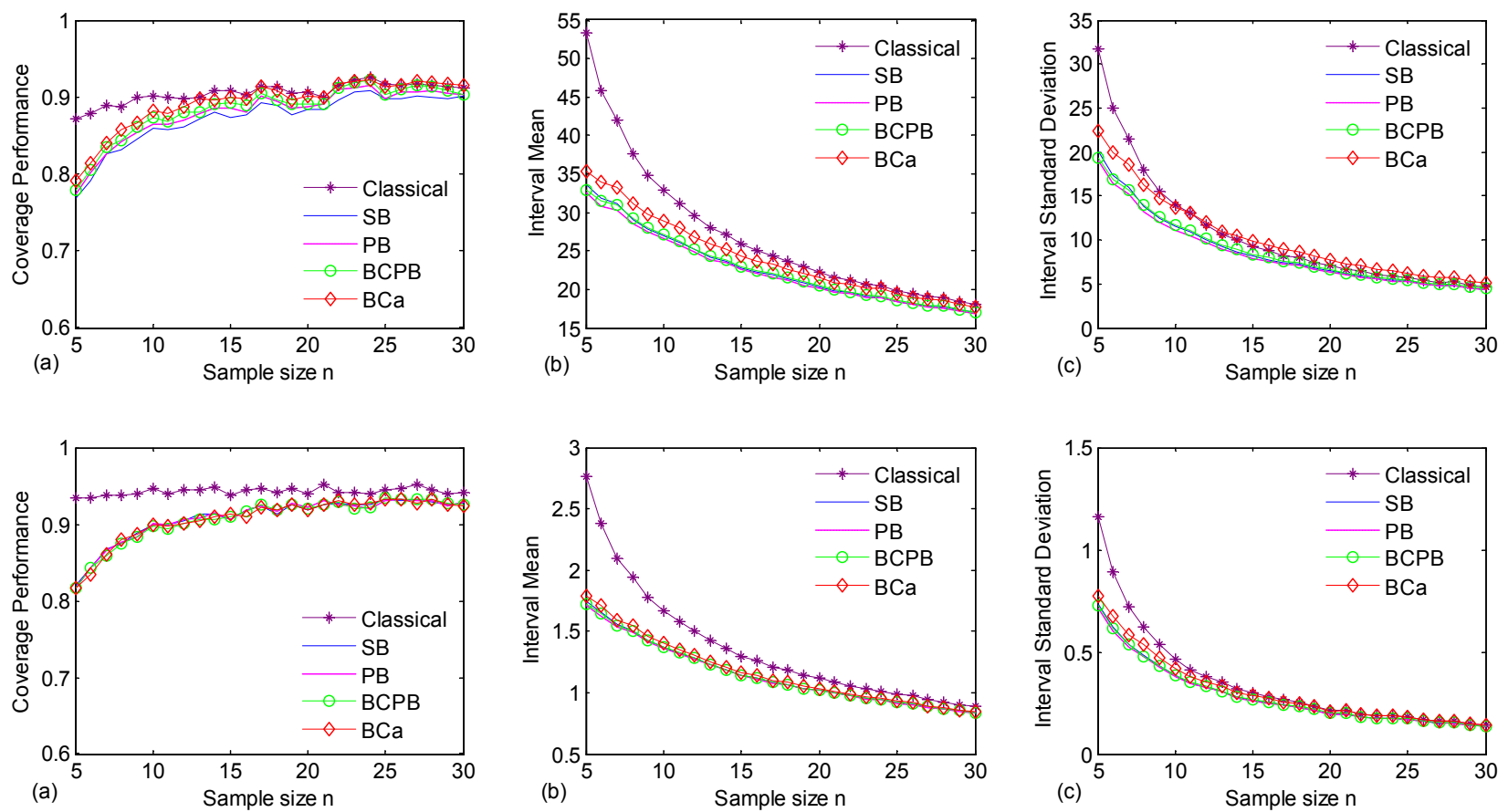

Figure 2. Coverage performance, interval mean and interval standard deviation vs. sample size under Weibull $(\alpha=10, \beta=$ $25)$ and Weibull $(\alpha=25, \beta=25)$.
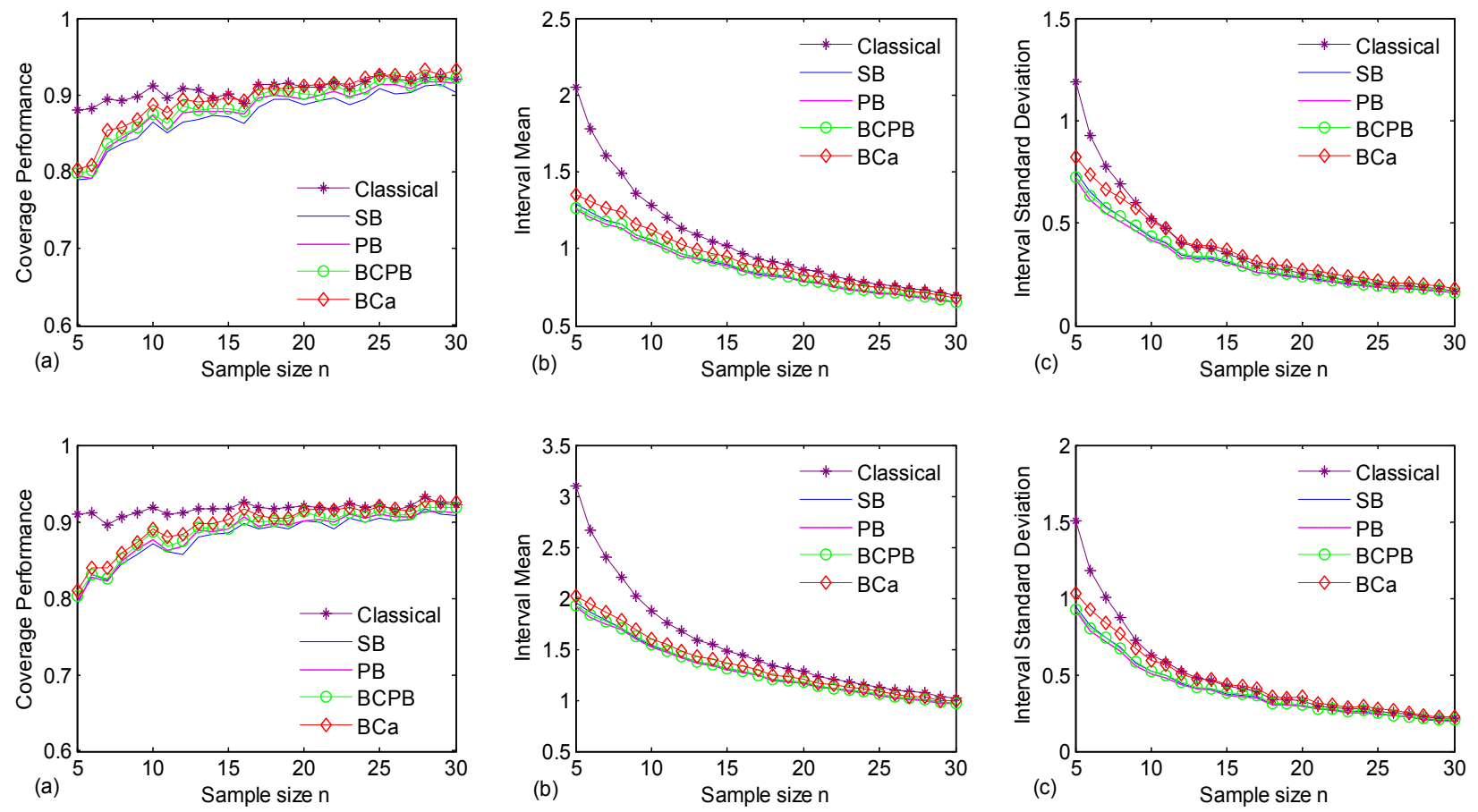

Figure 3. Coverage performance, interval mean and interval standard deviation vs. sample size under Gamma $(\alpha, \beta)=(1.0$, $1.0)$ and Gamma $(\alpha=2.0, \beta=1.0)$. 

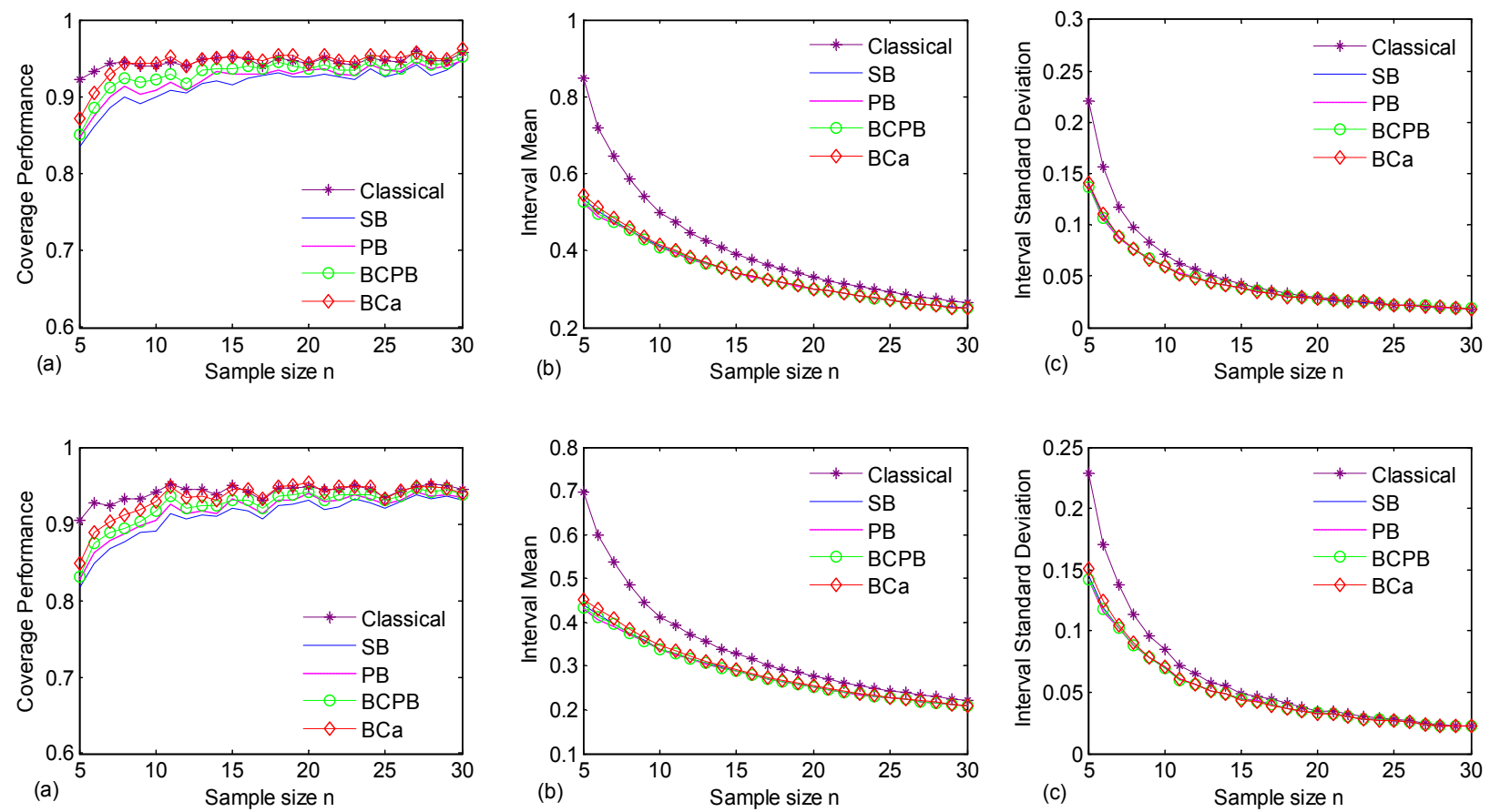

Figure 4. Coverage performance, interval mean and interval standard deviation vs. sample size under $\operatorname{Beta}(\alpha=0.5, \beta=0.5)$ and Beta $(\alpha=1.0, \beta=0.5)$.

than the remaining three bootstrap confidence intervals for a non-normal dataset in terms of coverage performance. When sample size is greater than or equal to 15 , the coverage performance of BCa confidence interval is approximately 0.90 . Therefore, this study recommends $\mathrm{BCa}$ confidence interval to quantify the uncertainty of the emission estimates with minimum sample size 15 .

\section{Case Study of Emission Data from Oil and Natu- ral Gas Industry}

This case study demonstrated the effectiveness of the proposed procedure based on emission data taken from the American Carbon Registry for emission reduction measurement and monitoring methodology for conversion of high-bleed pneumatic controllers in oil and natural gas systems (American Carbon Registry, 2010). The emission data set contained a total of 35 measurements from continuous high-bleed pneumatic controllers of two manufacturers (Cemco and Invalco). The results of the $t$-test for independent two samples showed that there is a significant difference between the population means of Cemco and Invalco controllers (American Carbon Registry, 2010). The detailed procedure of data collection is given in the American Carbon Registry. Pneumatic controllers powered by the pressurized natural gas are used to regulate the process variables such as pressure, flow rate and liquid level. As part of normal operations, pneumatic controllers release the methane emissions to the atmosphere from natural gas industry. Pneumatic controllers can be designed at both high and low-bleed rate. The conversion of high-bleed controller in oil and natural gas industry can re- duce these emissions. The replacement of high-bleed with lowbleed depends on the baseline monitoring methodology which describes the baseline measurement and monitoring procedures. The baseline scenario is the continued use of high-bleed pneumatic controllers. Baseline emissions are comprised of the natural gas vented to the atmosphere from these high-bleed controllers. Uncertainties related to the estimates of methane emission reductions from high-bleed pneumatic controller are to be quantified.

The calculation of the uncertainty of emission rate for baseline emission can be clearly performed based on statistical assumptions. The assumptions are that the error in the emission rate is normally distributed and the errors in the parameter estimates are independent. The classical confidence intervals for emission rate were computed based on these above assumptions. Alternative calculation method is, non-parametric bootstrap intervals, were used, when emission data is not normal.

Table 2 summarizes the high-bleed rates (i.e., emissions data) taken from the direct measurement of 35 controllers, which are from two manufactures: Cemco and Invalco. The mean bleed rate is 518.94 scfd for Cemco controllers and is 628.86 scfd for Invalco controllers. The $95 \%$ confidence intervals are used to quantify the uncertainty of the mean bleed rate of Cemco and Invalco controllers. Uncertainty of the mean bleed rate of various controllers was quantified by using two samples from two oil and natural gas systems. Based on the procedure in Section 3, Steps (iii) (v) were performed without any distribution assumption to construct the four bootstrap confidence intervals. Tables 3 and 4 summarize the classical confidence interval and four bootstrap confidence intervals for the mean of the two emi- 

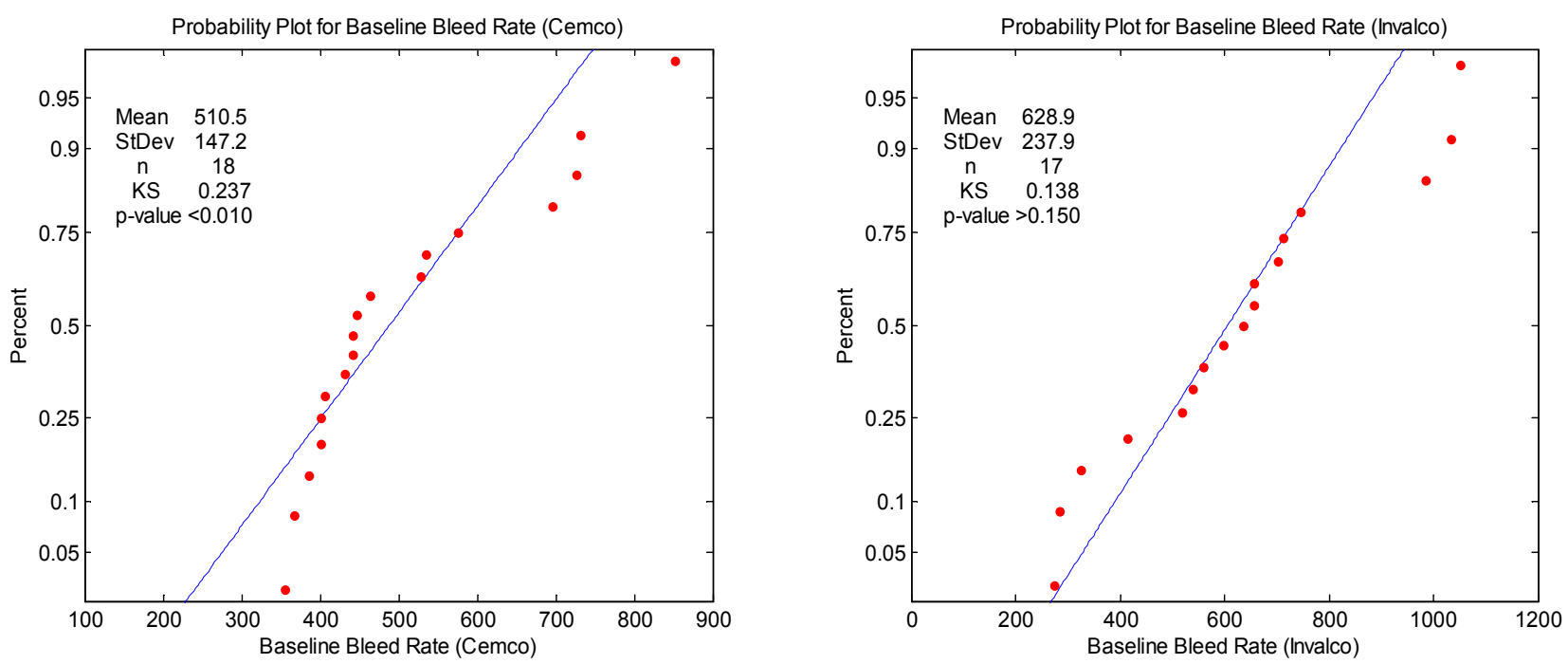

Figure 5. Probability plots for baseline bleed rate of emission Data from Oil and Natural Gas Systems for Cemco and Invalco.

ssion datasets, respectively. With respect to the interval width, the confidence intervals for the mean of emission data from the bootstrap methods are shorter than the classical method. The four bootstrap intervals provide satisfactory coverage percentage under three non-normal distributions and $\mathrm{BCa}$ interval provides slightly higher coverage percentage with smaller interval width than other three confidence intervals. Therefore, the uncertainty estimate for high-bleed rates from BCa method are utilized and they are approximately \pm 64.55 (Cemco) and \pm 108.64 (Invalco), respectively.

Moreover, the probability plots in Figure 5 indicated that the emissions data of Cemco manufacturer is not normal $(\mathrm{KS}=$

Table 2. High-Bleed Pneumatic Controller Emission Data

\begin{tabular}{ll}
\hline \multicolumn{2}{l}{ Baseline bleed rate, scfd, Manufacturer } \\
\hline Cemco $(\mathrm{n}=18)$ & Invalco $(\mathrm{n}=17)$ \\
\hline 367 & 518.1 \\
432 & 274.3 \\
528 & 655 \\
732 & 1052.2 \\
463.2 & 597.6 \\
576 & 559.2 \\
696 & 415.2 \\
535.2 & 636 \\
726.7 & 744 \\
851.5 & 540 \\
401.3 & 285 \\
386 & 984 \\
441.9 & 1034 \\
355.6 & 712 \\
401.3 & 657 \\
447 & 703 \\
441.9 & 324 \\
406.4 & \\
\hline
\end{tabular}

0.237 and $p$-value $<0.010)$. Also, the emissions data of Invalco manufacturer is normal ( $\mathrm{KS}=0.138$ and $\mathrm{p}$-value $>0.150)$. Obviously, according to Tables 3 and 4, when the sample size is small, bootstrap confidence intervals are more applicable than the classical confidence interval in both circumstances, regardless of the distribution is normal or non-normal.

\section{Conclusions}

In general, the classical method is used to construct the confidence interval for quantifying the uncertainty of GHG emission estimates when sample size is sufficiently large or the distribution is normal. However, using the classical method to construct confidence interval for quantifying the uncertainty of GHG emission estimates may lead to a significant bias when the sample size is small or the distribution is non-normal. In practice, obtaining a large emission dataset is difficult and fitted a distribution to emission data of small sample size may not be accurate. Therefore, this study quantifies the uncertainty of emission estimates for non-normal distributions (namely, Weibull, Gamma and Beta) using non-parametric bootstrap method. Sensitivity analysis with various combinations of sample size and parameter values was performed to examine the behavior of four bootstrap confidence intervals (i.e. $\mathrm{SB}, \mathrm{PB}, \mathrm{BCPB}$ and $\mathrm{BCa}$ ) and also the classical confidence interval. Three performance indices were utilized to examine the accuracy and precision of the uncertainty for various interval estimations. Simulation results indicated that the sample size significantly impacts the performance of the confidence intervals for non-normal distributions. A large sample size always increases the coverage performance, reduces the interval mean, and decreases the interval standard deviation of the bootstrap confidence intervals. Compared with the classical confidence interval, bootstrap confidence intervals have smaller interval mean and smaller interval standard deviation for small sample size $(n \leq 30)$ under nonnormal distributions. Although all four bootstrap confidence 
Table 3. Confidence Intervals for Emission Data of Cemco (Baseline bleed rate, scfd)

\begin{tabular}{lllllll}
\hline \multirow{2}{*}{ Method } & \multicolumn{2}{l}{$95 \%$ Confidence Interval } & \multirow{2}{*}{ Interval Length } & & \multicolumn{2}{l}{ Coverage Performance } \\
\cline { 2 - 3 } & Lower limit & Upper Limit & & Gamma & Weibull & Beta \\
\hline Classical & 437.30 & 583.70 & 146.40 & 132.41 & 0.9520 & 0.9445 \\
SB & 443.97 & 576.38 & 132.34 & 0.9310 & 0.9245 & 0.9535 \\
PB & 447.01 & 579.35 & 135.33 & 0.9335 & 0.9250 & 0.9255 \\
BCPB & 452.20 & 587.53 & 129.10 & 0.9340 & 0.9225 & 0.9310 \\
BCa & 456.09 & 585.19 & 0.9365 & 0.9270 & 0.9315 \\
\hline
\end{tabular}

Table 4. Confidence Intervals for Emission Data of Invalco (Baseline bleed rate, scfd)

\begin{tabular}{lllllll}
\hline \multirow{2}{*}{ Method } & \multicolumn{2}{l}{$95 \%$ Confidence Interval } & \multirow{2}{*}{ Interval Length } & & \multicolumn{2}{l}{ Coverage Performance } \\
\cline { 2 - 3 } & Lower limit & Upper Limit & & Gamma & Weibull & Beta \\
\hline Classical & 506.56 & 751.15 & 244.59 & 219.80 & 0.9435 & 0.9470 \\
SB & 521.90 & 741.69 & 223.03 & 0.9155 & 0.9250 & 0.9565 \\
PB & 520.23 & 743.26 & 217.64 & 0.9165 & 0.9260 & 0.9310 \\
BCPB & 526.51 & 744.16 & 217.27 & 0.9180 & 0.9275 & 0.9355 \\
BCa & 528.54 & 745.81 & 0.9215 & 0.9315 & 0.9375 \\
\hline
\end{tabular}

intervals display a similar trend in terms of coverage performance, the BCa confidence interval performs better than the remaining three bootstrap confidence intervals for non-normal distributions, especially when the sample size is 15 or more. When the sample size exceeds 30 , either the classical confidence interval or bootstrap confidence intervals may be used regardless of whether the distribution is normal or non-normal. A case study with small sample size also showed that BCa confidence interval performs better than other intervals with shorter interval length. The non-parametric bootstrap confidence intervals for quantifying the uncertainty of the emission estimates can be obtained quickly by using computer software packages.

\section{References}

American Carbon Registry (2010). Emission Reduction Measurement and Monitoring Methodology for Conversion of High-Bleed Pneumatic Controllers in Oil and Natural Gas Systems, Verdeo Group, Inc. and Devon Energy Corporation.

Chou, C.Y., Lin, Y.C., Chang, C.L., and Chen, C.H. (2006). On the bootstrap confidence intervals of the process incapability index $\mathrm{C}_{\mathrm{pp}}$. Reliab. Eng. Syst. Saf., 91(4), 452-459. http://dx.doi.org/10.1016/j. ress.2005.03.004

Chu, Y.K., and Ke, J.C. (2006). Confidence intervals of mean response time for an $\mathrm{M} / \mathrm{G} / 1$ queueing system: Bootstrap simulation. Appl. Math. Computation, 180(1), 255-263. http://dx.doi.org/10.10 16/j.amc.2005.11.145

Efron, B. (1979). Bootstrap method: Another look at the Jackknife. Anal. Stat., 7(1), 1-26. http://dx.doi.org/10.1214/aos/1176344552

Efron, B. (1981). Nonparameter estimates of standard error: The jackknife, the bootstrap and other sampling methods. Biometrika, 68(3), 589-599. http://dx.doi.org/10.1093/biomet/68.3.589

Efron, B. (1982). The Jackknife, the bootstrap and other resampling plans, CBMS-NSF Regional Conference Series in Applied Mathematics, Philadelphia, PA USA. http://dx.doi.org/10.1137/1.97816 11970319

Efron, B. (1987). Better bootstrap confidence intervals. J. Am. Stat.
Assoc., 82(397), 171-185. http://dx.doi.org/10.1080/01621459.1987. 10478410

Efron, B., and Gong, G. (1983). A leisurely look at the bootstrap, the Jackknife, and cross-validation. Am. Statistician, 37(1), 36-48. http://dx.doi.org/10.2307/2685844

Efron, B., and Tibshirani, R. (1986). Bootstrap methods for standard efforts, confidence intervals, and other measures of statistical accuracy. Stat. Sci., 1(1), 54-75. http://dx.doi.org/10.1214/ss/1177013 815

Efron, B., and Tibshirani, R. (1993). An Introduction to the Bootstrap, Champman \& Hall, London, U.K. http://dx.doi.org/10.1007/978-14899-4541-9

EMEP/EEA (2013). Air Pollutant Emission Inventory Guidebook, Part A: General guidance chapters -- Chapter 5: Uncertaintie. http://www.eea.europa.eu/publications/emep-eea-guidebook-2013.

Erickson, P., Chandler, C., and Lazarus, M. (2012). Reducing Greenhouse Gas Emissions Associated with Consumption: A Methodology for Scenario Analysis, Stockholm Environment Institute Working Paper No. 2012-05.

Frey, H.C., and Bammi, S. (2002). Quantification of variability and uncertainty in lawn and garden equipment $\mathrm{NO}_{\mathrm{x}}$ and total hydrocarbon emission factors. J. Air Waste Manage. Assoc., 52(4), 435-448. http://dx.doi.org/10.1080/10473289.2002.10470792

Frey, H.C., and Li, S. (2003). Methods for quantifying variability and uncertainty in AP-42 emission factors: Case studies for natural gas-fueled engines. J. Air Waste Manage. Assoc., 53 (12), 14361447. http://dx.doi.org/10.1080/10473289.2003.10466317.

Frey, H.C., and Rhodes, D.S. (1996a). Characterization and simulation of uncertainty frequency distributions: Effects of distribution choice, variability, uncertainty and parameter dependence. Hum. Ecol. Risk Assess., 4(2), 428-468. http://dx.doi.org/10.1080/1080 7039891284406

Frey, H.C., and Rhodes, D.S. (1996b). Characterizing, simulating, and analyzing variability and uncertainty: An illustration of methods using an air toxics emissions example. Hum. Ecol. Risk Assess., 2(4), 762-797. http://dx.doi.org/10.1080/10807039609383650

Frey, H.C., and Zhao, Y. (2004). Quantification of variability and uncertainty for air toxic emission inventories with censored emission factor data. Environ. Sci. Technol., 38(22), 6094-6100. http://dx.doi.org/10.1021/es035096m 
Frey, H.C., Bharvirkar, R., and Zheng, J. (1999). Quantitative Analysis of Variability and Uncertainty in Emission Estimation, Prepared by North Carolina State University for Office of Air Quality Planning and Standards U.S. Environmental Protection Agency, Research Triangle Park, NC.

GHG Protocol (2003). WRI, GHG. Protocol guidance on uncertainty assessment in GHG inventories and calculating statistical parameter uncertainty: Short Guidance for Calculating Measurement and Estimation Uncertainty for GHG Emissions, World Resources Institute: Washington DC. http://www.ghgprotocol.org/files/ghgp/ tools/ghg-uncertainty.pdf.

IPCC (2001). Good practice guidance and uncertainty management in national greenhouse gas inventories, in J. Penman et al. (Eds.), IPCC National Greenhouse Gas Inventories Programme, Technical Support Unit, Hayama, Japan.

IPCC (2006). IPCC guidelines for national greenhouse gas inventories, in H.S. Eggleston, L. Buendia, K. Miwa, T. Ngara and K. Tanabe (Eds.), Intergovernmental Panel on Climate Change IPCC, IPCC/OECD/IEA, Hayama, Japan.

IPCC (2007). Climate change 2007: Impacts, adaptation and vulnerability, in M.L. Parry, O.F. Canziani, J.P. Palutikof, P.J. Van Der Linden and C.E. Hanson (Eds.), Contribution of Working Group II to the Fourth Assessment Report of the Intergovernmental Panel on Climate Change, Cambridge University Press, Cambridge

Kumar, S., Himanshu S.K., and Gupta K.K. (2012). Effect of global warming on mankind -- A review. Int. Res. J. Environment Sci. $1(4), 56-59$.

Kyselý, J. (2010). Coverage probability of bootstrap confidence intervals in heavy-tailed frequency models, with application to precipitation data. Theor. Appl. Climatol., 101(3), 345-361. http://dx. doi.org/10.1007/s00704-009-0190-1

Millar, N., Robertson, G.P., Diamant, A., Gehl, R.J., Grace, P.R., and Hoben, J.P. (2012). Methodology for Quantifying Nitrous Oxide $\left(\mathrm{N}_{2} \mathrm{O}\right)$ Emissions Reductions by Reducing Nitrogen Fertilizer Use on Agricultural Crops, American Carbon Registry, Winrock International, Little Rock, Arkansas.

Monni, S., and Syri, S. (2004). Uncertainties in the Finnish 2002 Greenhouse Gas Emission Inventory, Espoo, VTT Tiedotteita -Research Notes 2209.

Moore, D.S., McCabe, G.P., and Craig, B.A. (2008). Introduction to the Practice of Statistics (6th Edition), W.H. Freeman, New York.

Mudhoo, A., Sewhoo, B., Mohee, R., and Gurjar, B. R. (2013). Greenhouse gas emissions reductions from in-situ aeration in a landfill: A multi-parameter sensitivity analysis approach, J. Environ. Inf., 22(2), 78-91. http://dx.doi.org/10.3808/jei.201300247

Niwitpong, S., and Kirdwichai, P. (2008). Adjusted bonett confidence interval for standard deviation of non-normal distributions. Thail. Statistician, 6(1), 1-6.

Pinto, J., Bonacic, C.W.C., Romero, J., and Lubroth, J. (2008). Climate change and animal diseases in South America. Rev. Sci. Tech., 27(2), 599-613.
Rhodes, D.S., and Frey, H.C. (1997). Quantification of variability and uncertainty in AP-42 emission factors using bootstrap simulation, Emission Inventory: Planning for the Future (held October 28-30 in Research Triangle Park, NC), Air and Waste Management Association, Pittsburgh, Pennsylvania, 147-161.

Riebeek, H. (2010). Global Warming, NASA Earth Observatory. http://earthobservatory.nasa.gov/Features/GlobalWarming/global_ warming_2007.pdf

Rypdal, K., and Winiwarter, W. (2001). Uncertainties in greenhouse gas emission inventories -- evaluation, comparability and implications. Environ. Sci. \& Policy, 4(2-3), 107-116. http://dx.doi.org/10. 1016/S1462-9011(00)00113-1

Shen, Y.S., Tan, Z.F., Shen, X.L., Bai, J.J., Li, Q.Z., and Wang, S. (2012). Study of energy saving and emission reduction based on the OLAP multi-indicator relational model, J. Environ. Inf., 20(2), 115-122. http://dx.doi.org/10.3808/jei.201200225

Tong, L.I., Chang, C.W., Jin, S.E., and Saminathan, R. (2012). Quantifying uncertainty of emission estimates in National Greenhouse Gas Inventories using bootstrap confidence intervals. Atmos. Environ., 56, 80-87. http://dx.doi.org/10.1016/j.atmosenv.2012.03.063

Tong, L.I., Chen, H.T., and Tai, Y.F. (2008). Constructing BCa bootstrap confidence interval for the difference between two non- normal process capability indices C $_{\text {Npmk. }}$ Qual. Eng., 20(2), 209-220. http://dx.doi.org/10.1080/08982110701724348

UNFCCC (1997). Kyoto protocol to the United Nations framework convention on climate change. in Proc. of the Third Session of Conference of the Parties (Report), Kyoto, 1 - 11 December 1997 (Addendum). Part Two: Action taken by the Conference of the Parties at its Third Session. Decision 1/CP.3, Annex (FCCC/CP/1997/7/ Add.1.) UNFCCC, Bonn, Germany.

Watson, C., Mourato, S., and Milner-Gulland, E.J. (2013). Uncertain emission reductions from forest conservation: REDD in the Bale Mountains, Ethiopia. Ecol. Soc., 18(3), 6. http://dx.doi.org/10.5751/ ES-05670-180306

Zhao, Y., and Frey, H.C. (2003). Quantification of uncertainty and variability for air toxic emission factor data sets containing nondetects, in Proc. of Annual Meeting of the Air \& Waste Management Association, 53(12), pp. 1436-47.

Zhao, Y., and Frey, H.C. (2006). Uncertainty for data with non-detects: Air toxix emission from combustion. Hum. Ecol. Risk Assess. Int. J., 12(6), 1171-1191. http://dx.doi.org/10.1080/108070306009771 78

Zhao, Y., Nielsen, C.P., Lei, Y., McElroy, M.B., and Hao, J. (2011). Quantifying the uncertainties of a bottom-up emission inventory of anthropogenic atmospheric pollutants in China. Atmos. Chem. Phys., 11, 2295-2308. http://dx.doi.org/10.5194/acp-11-2295-2011

Zheng, J. (2002). Quantification of Variability and Uncertainty in Emission Estimation: General Methodology and Software Implementation, Ph.D. Dissertation, North Carolina State University, USA. 